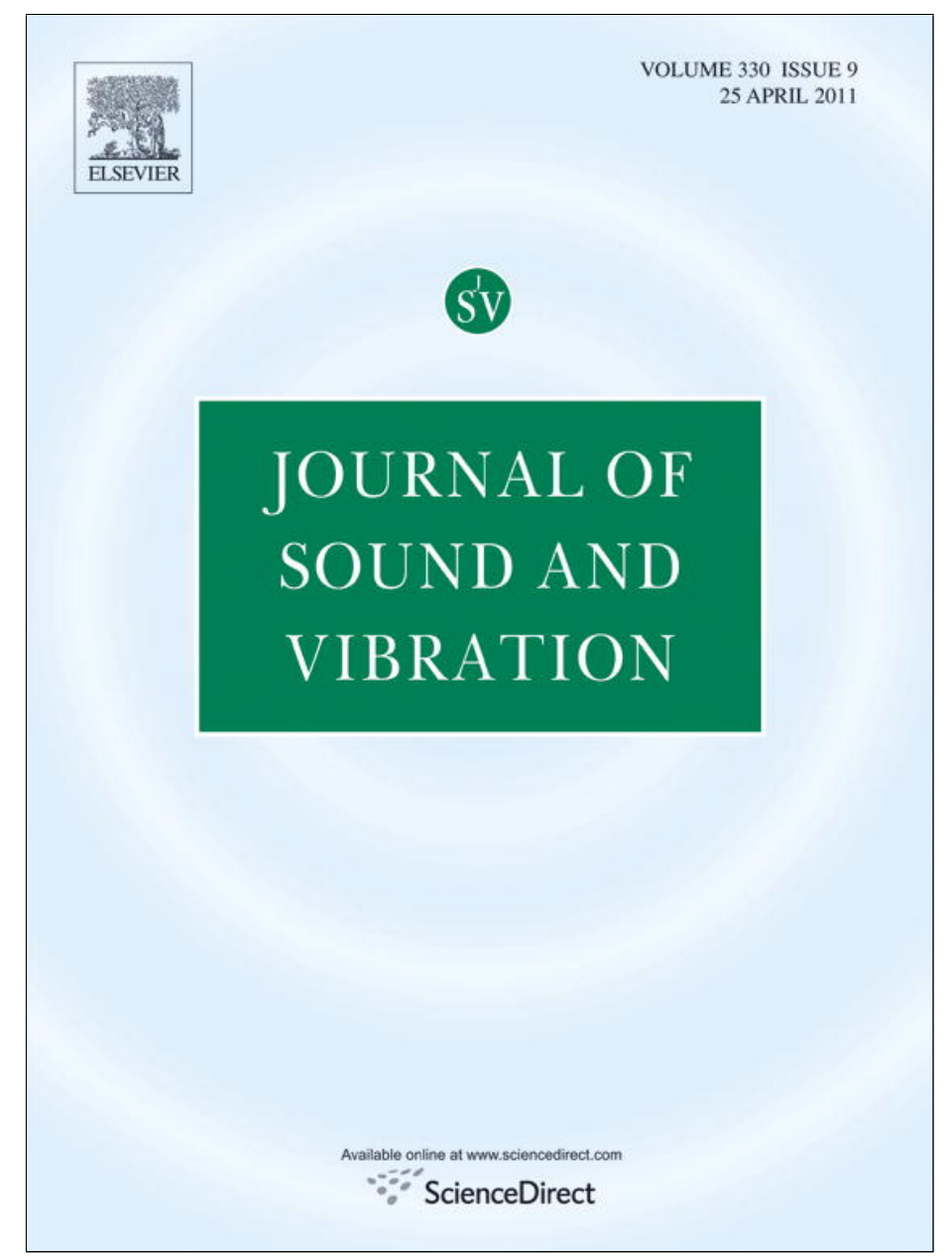

This article appeared in a journal published by Elsevier. The attached copy is furnished to the author for internal non-commercial research and education use, including for instruction at the authors institution and sharing with colleagues.

Other uses, including reproduction and distribution, or selling or licensing copies, or posting to personal, institutional or third party websites are prohibited.

In most cases authors are permitted to post their version of the article (e.g. in Word or Tex form) to their personal website or institutional repository. Authors requiring further information regarding Elsevier's archiving and manuscript policies are encouraged to visit:

http://www.elsevier.com/copyright 


\title{
Perturbation theory and the Rayleigh quotient
}

\author{
K.T. Chan ${ }^{\text {a }}$, N.G. Stephen ${ }^{\mathrm{b}}$, K. Young ${ }^{\mathrm{c}, *}$ \\ a Department of Mechanical Engineering, The Hong Kong Polytechnic University, Hong Kong, People's Republic of China \\ b School of Engineering Sciences, Mechanical Engineering, University of Southampton, Highfield, Southampton SO17 1BJ, UK \\ c Department of Physics, The Chinese University of Hong Kong, Hong Kong, People's Republic of China
}

\section{A R T I C L E I N F O}

\section{Article history:}

Received 27 July 2010

Received in revised form

30 October 2010

Accepted 1 November 2010

Handling Editor: S. Ilanko

Available online 19 November 2010

\begin{abstract}
A B S T R A C T
The characteristic frequencies $\omega$ of the vibrations of an elastic solid subject to boundary conditions of either zero displacement or zero traction are given by the Rayleigh quotient expressed in terms of the corresponding exact eigenfunctions. In problems that can be analytically expanded in a small parameter $\varepsilon$, it is shown that when an approximate eigenfunction is known with an error $O\left(\varepsilon^{N}\right)$, the Rayleigh quotient gives the frequency with an error $O\left(\varepsilon^{2 N}\right)$, a gain of $N$ orders. This result generalizes a well-known theorem for $N=1$. A non-trivial example is presented for $N=4$, whereby knowledge of the 3rd-order eigenfunction (error being 4th order) gives the eigenvalue with an error that is 8th order; the 6th-order term thus determined provides an unambiguous derivation of the shear coefficient in Timoshenko beam theory.
\end{abstract}

(c) 2010 Elsevier Ltd. All rights reserved.

\section{Introduction}

The determination of the characteristic frequencies $\omega$ of vibrations in a solid is a central issue in the theory of linear elasticity. Approximate methods include perturbation expansions in a small parameter $\varepsilon$ and the variational method. This paper deals with a synergy between the two: if a perturbed eigenfunction with error $O\left(\varepsilon^{N}\right)$ is used judiciously in a variational functional, the eigenvalue can be evaluated with an error $O\left(\varepsilon^{2 N}\right)$ - a gain of $N$ orders "for free". To be specific, vibrations in an elastic solid are described by the displacement field $\xi(\boldsymbol{r}, t)$, where $t$ is the time, $\boldsymbol{r}=\left(r_{1}, r_{2}, r_{3}\right)=(x, y, z)$ is the position and $\xi=\left(\xi_{1}, \xi_{2}, \xi_{3}\right)=(u, v, w)$ is the displacement. Linearized displacements satisfy the time-dependent equation of motion (1) in a certain volume $\mathcal{V}$ and either one of the conditions (2) or (3) on its boundary $\mathcal{S}$ :

$$
\begin{gathered}
\rho \frac{\partial^{2} \xi_{i}}{\partial t^{2}}=\partial_{j}\left[C_{i j k \ell}\left(\partial_{k} \xi_{\ell}\right)\right], \\
\xi_{i}=0, \\
n_{j} C_{i j k \ell}\left(\partial_{k} \xi_{\ell}\right)=0,
\end{gathered}
$$

for $i=1,2,3$, where $\rho(\boldsymbol{r})$ is the density, $C_{i j k \ell}(\boldsymbol{r})$ are the stiffness constants (not necessarily isotropic) and $n_{j}$ are the components of the unit normal to $\mathcal{S}$. The convention of summing over repeated indices is adopted. The position-dependence of $\rho, C_{i j k \ell}$ and $\xi$ will henceforth not be displayed. The possibility is allowed that (2) holds on one part of $\mathcal{S}$ and (3) holds on another. Normal modes $\propto \cos \omega t$ satisfy the eigenvalue equation with eigenvalue $\lambda=\omega^{2}$ :

$$
-\partial_{j}\left[C_{i j k \ell}\left(\partial_{k} \xi_{\ell}\right)\right]=\lambda \rho \xi_{i}
$$

\footnotetext{
* Corresponding author. Fax: +852 26035252 .

E-mail addresses: mmktchan@polyu.edu.hk (K.T. Chan), n.g.stephen@soton.ac.uk (N.G. Stephen), kyoung@cuhk.edu.hk (K. Young).
} 
The eigenvalue can be expressed exactly in terms of the Rayleigh quotient (RQ) [1,2]:

$$
\lambda=Q[\xi] \equiv \frac{V[\xi]}{T[\xi]}
$$

where $V / 2$ and $\omega^{2} T / 2$ are essentially the potential and kinetic energies:

$$
\begin{gathered}
V[\xi]=\int C_{i j k \ell}\left(\partial_{i} \xi_{j}\right)\left(\partial_{k} \xi_{\ell}\right) \mathrm{d} \mathcal{V}, \\
T[\xi]=\int \rho \xi_{j} \xi_{j} \mathrm{~d} \mathcal{V},
\end{gathered}
$$

and $\xi$ is the corresponding exact eigenfunction. It will be everywhere understood that volume integrals are carried out over $\mathcal{V}$ and surface integrals over $\mathcal{S}$. To prove (5), integrate (6a) by parts and notice that surface terms vanish by (2) or (3); then $V$ is seen to be $\lambda T$ by (4).

The RQ leads to the variational principle [3]: the solutions to (4) are given by $\delta Q[\xi]=0$ over the linear space $\mathcal{L}$ of all $\xi$ satisfying the boundary conditions. This is readily proved by noticing that, since both $V$ and $T$ are quadratic in $\xi$, one can equivalently vary $V$ under the constraint $T=1$ implemented by a Lagrange multiplier $\lambda$ :

$$
\delta(V-\lambda T)=0,
$$

and $\lambda$ will turn out to be the eigenvalue denoted by the same symbol.

The object of the present paper, however, is not to consider $Q$ over all $\xi \in \mathcal{L}$, but for those $\xi$ close to the exact eigenfunction. To formalize the idea in a perturbation scheme, let $C_{i j k \ell}$ and/or $\rho$ be described by a small parameter $\varepsilon$ :

$$
C_{i j k \ell}=C_{i j k \ell}(\varepsilon ; \boldsymbol{r}), \quad \rho=\rho(\varepsilon ; \boldsymbol{r}),
$$

with an analytic limit as $\varepsilon \rightarrow 0 ; C_{i j k \ell}$ and $\rho$ need not be linear in $\varepsilon$. The exact eigenfunction $\xi$ is expanded in powers of $\varepsilon$ :

$$
\xi_{i}=\sum_{n=0}^{\infty} \varepsilon^{n} \xi_{i}^{(n)}
$$

For some $N \geq 1$, divide $\xi$ into the $n<N$ terms (denoted as low-order or $L$ ) and the $n \geq N$ terms (denoted as high-order or $H$ ):

$$
\begin{gathered}
\xi_{i}=\xi_{i}^{L}+\xi_{i}^{H}, \\
\xi_{i}^{L}=\sum_{n=0}^{N-1} \varepsilon^{n} \xi_{i}^{(n)}, \quad \xi_{i}^{H}=\sum_{n=N}^{\infty} \varepsilon^{n} \xi_{i}^{(n)},
\end{gathered}
$$

so that $\xi_{i}^{L}$ is an approximation to $\xi_{i}$ with an error that is $O\left(\varepsilon^{N}\right)$. In all these formulas, the expansions in powers of $\varepsilon$ are meant to be asymptotic, with no claims on convergence. In other words, the attention is on the $\varepsilon \rightarrow 0$ behaviour of the series truncated at fixed $N$, and not on the $N \rightarrow \infty$ limit at fixed $\varepsilon$.

Our key result is: this approximation, when used in the RQ yields the eigenvalue with an error that is $O\left(\varepsilon^{2 N}\right)$. More precisely, we shall prove the following theorem:

$$
\lambda \approx Q\left[\xi^{L}\right]
$$

where the notation $A \approx B$ shall mean $A-B=O\left(\varepsilon^{2 N}\right)$.

It is well known that RQ allows one order to be gained [2]: an approximate eigenfunction with an error of $O\left(\varepsilon^{N}\right)$ gives the eigenvalue with an error of $O\left(\varepsilon^{N+1}\right)$; in the familiar $N=1$ case, the unperturbed eigenfunction with error $O(\varepsilon)$ gives the firstorder eigenvalue with error $O\left(\varepsilon^{2}\right)[1,4]$. However, it is less obvious, and indeed surprising at first sight, that $N$ orders can be gained "for free". The analogous theorem for the Schrödinger equation is known, but not prominently, being contained for example in a footnote [5], which also cites a discussion about partitioning the Hilbert space into two parts coupled by a parameter $\varepsilon$ [6]. Adams [7] also gives an expression for the eigenvalue with an error of $O\left(\varepsilon^{2 N}\right)$ in terms of the eigenfunction with an error of $O\left(\varepsilon^{N}\right)$, though not in the form of an expectation value (the quantum-mechanical analog of the RQ) and therefore taking a more cumbersome form. The case of elasticity considered here is more complicated, not only because of the tensorial nature, but also because the boundary condition (3) can link different orders in $\varepsilon$. The theorem is powerful (for $N>1$ ), but has hitherto received little attention, probably because it is in general difficult to obtain the eigenfunction to many orders; however, Section 3 shows an $N=4$ example in elasticity related to Timoshenko beam theory, where a computable 3rd-order eigenfunction [error of $O\left(\varepsilon^{4}\right)$ ] allows the eigenvalue to be determined with an error of $O\left(\varepsilon^{8}\right)$; it turns out that the 6th-order term, which can thus be obtained, is physically non-trivial.

\section{Proof}

In obvious notation, both the numerator $V$ and the denominator $T$ are the sum of $L L, L H$ and $H H$ terms. The last of these are negligible, being $O\left(\varepsilon^{2 N}\right)$, so the key result requires only a proof that the LH cross-terms do not contribute. The proof is in fact not that much different from the familiar case of $N=1$. 


\subsection{Denominator}

Start with

$$
\begin{gathered}
\int \rho \xi_{i}^{L} \xi_{i}^{L} \mathrm{~d} \mathcal{V} \equiv N_{L}>0, \\
\int \rho \xi_{i}^{L} \xi_{i}^{H} \mathrm{~d} \mathcal{V}=N_{L} f(\varepsilon)=O\left(\varepsilon^{N}\right),
\end{gathered}
$$

where (13a) is just a normalization. There is the freedom to make an $\varepsilon$-dependent change of normalization:

$$
\begin{gathered}
\xi \mapsto[1-f(\varepsilon)] \xi, \\
\xi^{L} \mapsto \xi^{L}, \\
\xi^{H} \mapsto \xi^{H}-f(\varepsilon) \xi^{L}, \\
\int \rho \xi_{i}^{L} \xi_{i}^{H} \mathrm{~d} \mathcal{V} \mapsto 0 .
\end{gathered}
$$

Thus, we can assume that $\xi^{L}$ and $\xi^{H}$ are orthogonal in this sense, and

$$
T[\xi] \approx T\left[\xi^{L}\right] .
$$

\subsection{Numerator}

Similarly, in the numerator consider the cross-term:

$$
\int C_{i j k \ell}\left(\partial_{i} \xi_{j}^{L}\right)\left(\partial_{k} \xi_{\ell}^{H}\right) \mathrm{d} \mathcal{V} \approx \int C_{i j k \ell}\left(\partial_{i} \xi_{j}\right)\left(\partial_{k} \xi_{\ell}^{H}\right) \mathrm{d} \mathcal{V}=\int n_{k} C_{i j k \ell}\left(\partial_{i} \xi_{j}\right) \cdot \xi_{\ell}^{H} \mathrm{~d} \mathcal{S}-\int \partial_{k}\left[C_{i j k \ell}\left(\partial_{i} \xi_{j}\right)\right] \cdot \xi_{\ell}^{H} \mathrm{~d} \mathcal{V},
$$

where in the last step we have integrated by parts. In the surface integral, either the first or second factor vanishes if the boundary condition is respectively (3) or (2). By (4), the final volume integral in (16) becomes

$$
\lambda \int \rho \xi_{\ell} \xi_{\ell}^{H} \mathrm{~d} \mathcal{V}=\int \rho \xi_{\ell}^{L} \xi_{\ell}^{H} \mathrm{~d} \mathcal{V}+\int \rho \xi_{\ell}^{H} \xi_{\ell}^{H} \mathrm{~d} \mathcal{V}
$$

in which the first term vanishes by the orthogonality (14d) proved earlier, and the second term is $O\left(\varepsilon^{2 N}\right)$. This then completes the proof

$$
V[\xi] \approx V\left[\xi^{L}\right]
$$

and hence the key result (12).

\subsection{Discussion}

The key to the simplification lies in the orthogonality relationship (14d), which, order-by-order, consists of the $N$ conditions

$$
\sum_{k=0}^{j} \int \rho \xi_{i}^{(k)} \xi_{i}^{(N+j-k)} \mathrm{d} \mathcal{V}=0, \quad j=0, \ldots, N-1 .
$$

These are tedious especially for large $N$, but we only require that the orthogonalization can be carried out. If, as is usually the case, the orthogonalization is not actually carried out, this would amount to reversing the transformation (14a), under which

$$
V[\xi] \mapsto[1-f(\varepsilon)]^{-2} V[\xi], \quad T[\xi] \mapsto[1-f(\varepsilon)]^{-2} T[\xi],
$$

so that $L H$ cross-terms re-emerge in $V$ and $T$; as a result (15) and (18) would not hold. But because the ratio $Q[\xi]$ is unchanged under (20), the $L H$ terms must exhibit a cancellation when the quotient is evaluated, in a manner that might otherwise have been unexpected. Observation of such a cancellation in the example in the next section motivated the present work.

Several remarks should also be made in relation to the $\varepsilon$-dependence of $C_{i j k \ell}$ and $\rho$. First, they may link different orders in $\varepsilon$, so that $\xi^{L}$ and $\xi^{H}$ do not separately satisfy the equation of motion (1) or (4), nor the condition of zero surface traction (3); the latter property means, in particular, that $\xi^{L} \notin \mathcal{L}$. However, the two parts $\xi^{L}$ and $\xi^{H}$ do separately satisfy the condition of no displacement (2) on those parts of $\mathcal{S}$ where it is imposed for $\xi$-and this is precisely the condition needed above. Second, nowhere above do we expand $C_{i j k \ell}$ or $\rho$, e.g., as

$$
C_{i j k \ell}=\sum_{\alpha=0}^{J} \varepsilon^{\alpha} C_{i j k \ell}^{(\alpha)}, \quad \rho=\sum_{\beta=0}^{K} \varepsilon^{\beta} \rho^{(\beta)} .
$$


In this connection, note that $V\left[\xi^{L}\right]$ and $T\left[\xi^{L}\right]$ are defined with the full $C_{i j k \ell}$ and $\rho$ but a truncated $\xi$; as such they are positive definite if $C_{i j k \ell}$ and $\rho$ are physical and positive operators. In contrast, suppose a product (say for $T$ ) is expanded as a whole in powers of $\varepsilon$ :

$$
\begin{gathered}
T=\int \rho \xi_{i} \xi_{i} \mathrm{~d} \mathcal{V}=\sum_{n=0}^{\infty} \varepsilon^{n} T^{(n)}, \\
T^{(0)}=\int \rho^{(0)} \xi_{i}^{(0)} \xi_{i}^{(0)} \mathrm{d} \mathcal{V}, \\
T^{(1)}=\int\left[\rho^{(1)} \xi_{i}^{(0)} \xi_{i}^{(0)}+2 \rho^{(0)} \xi_{i}^{(0)} \xi_{i}^{(1)}\right] \mathrm{d} \mathcal{V},
\end{gathered}
$$

etc., and the series for $T$ truncated, then the result is not guaranteed to be positive definite.

It is necessary to point out the fallacies in two apparently simpler derivations. (a) Let the error in the eigenfunction be $O\left(\varepsilon^{N}\right)$ with some $N>1$, and define $\delta=\varepsilon^{N}$. Then it may seem that by the familiar theorem (namely the $N=1$ special case of the theorem discussed here), the error in the eigenvalue will be $O\left(\delta^{2}\right)=O\left(\varepsilon^{2 N}\right)$. This argument is invalid because it violates the implicit (but necessary) condition that functions are analytic in the small parameter-but here the eigenfunctions and eigenvalues are power series in $\delta^{1 / N}$. (b) One may also try to express the approximate eigenfunction, say $\xi^{L}$, as a linear superposition of the exact eigenfunctions, and adapt the derivation given by Rayleigh for finite degrees of freedom; see Eq. (2) in article 88 in Ref. [1]. However, because of the $\varepsilon$-dependence of $C_{i j k \ell}$ and the boundary condition (3), the space of functions $\mathcal{L}$ is $\varepsilon$-dependent, i.e., $\mathcal{L}=\mathcal{L}(\varepsilon)$. As a consequence, $\xi^{L} \notin \mathcal{L}(\varepsilon)$ and cannot be expressed as a linear superposition of the exact eigenfunctions. Incidentally, this problem does not occur in finite systems [1] or in simpler continuous analogs such as quantum mechanics [5-7]. The derivation presented in this paper avoids both of these difficulties.

\section{Example}

The ability to get $N$ more orders "for free" is powerful for large values of $N$, but would require knowledge of the eigenfunction in some manageable form with an error of $O\left(\varepsilon^{N}\right)$, which is often difficult in practice. This may be the reason why the theorem seems little known and seldom applied for $N>1$, even though the general proof is no more difficult than for $N=1$. An example is given in Appendix A in the context of a "toy" (but non-trivial) one-dimensional analog. More physically, this section sketches an $N=4$ example where it is possible to obtain an eigenfunction to 3rd order [i.e., with error of $O\left(\varepsilon^{4}\right)$ ], and consequently the eigenvalue with an error that is $O\left(\varepsilon^{8}\right)$. The 6th-order coefficient ${ }^{1}$ thus obtained is deeply related to Timoshenko beam theory [8], and provides one perspective to understand why it works so well.

The system of interest is the transverse flexural vibration of a uniform cylinder (beam) of length $L$ and characteristic lateral dimension $a$ (in the direction of vibration). In most engineering applications, $a / L \ll 1$ provides a small parameter for an asymptotic power-series expansion. Equivalently, consider an infinitely long cylinder (say oriented along the $z$ direction), in which there are sinusoidal waves say $\propto \sin q z$; one then seeks the dispersion relation $\omega=\omega(q)$. The case of a finite cylinder, say with hinged end-points, is recovered if $q$ is restricted to integral multiples of $\pi a / L$. Thus equivalently one can expand in $\varepsilon=q a$, or simply $q$, for example:

$$
\omega^{2}=A_{4} q^{4}+A_{6} q^{6}+\cdots
$$

The powers of $q$ emerge when $\partial_{z}$ operates on sinqz or cosqz, so to keep track of orders we can simply add a formal small parameter $\varepsilon$ to $\partial_{z}: \partial_{i} \mapsto \partial_{i}^{\perp}+\varepsilon \delta_{i 3} \partial_{z}$.

In (23), the leading $q^{4}$ term is just the classic Euler-Bernoulli theory, and depends on the cross-sectional shape only through a radius of gyration $r_{g}$. The next order, relevant for thicker and shorter cylinders, depends on the cross-section in a more complicated way. It might have been thought that $A_{6}$ requires a daunting calculation to 6 th (or at least 5 th) order. The import of the present paper is that one only need the eigenfunction up to 3rd order. The 0th, 1st and 2nd orders are easy, while the 3rd-order eigenfunction requires the solution of a partial differential equation in the two-dimensional crosssectional plane, giving rise to the Saint Venant flexure function [9]. The details of the straightforward but lengthy calculation will be given elsewhere; suffice it to say here that prior knowledge of the cancellation of higher-order terms makes the calculation much less onerous-in fact, one is then entitled to set the 4th-, 5th- and 6th-order terms in the eigenfunction to zero for this purpose.

Timoshenko beam theory [8] gives a correction to Euler-Bernoulli theory for shorter and thicker beams (i.e., $a / L$ not so small) and works very well in practice. The theory is based on a physical insight, through a shear coefficient $\kappa$ that describes the shear deformation of the cross-sectional plane as the beam is bent. The theory leads to a quadratic for $\omega^{2}$, which can likewise be developed into a power series in $q$, which (for the mode that satisfies $\omega \rightarrow 0$ as $q \rightarrow 0$ ) again has the form

$$
\omega^{2}=A_{4}^{\mathrm{T}} q^{4}+A_{6}^{\mathrm{T}}(\kappa) q^{6}+\cdots,
$$

\footnotetext{
${ }^{1}$ With $N=4$ and the error being $O\left(\varepsilon^{8}\right)$, the eigenvalue is known to 7th order, but in this example, odd orders vanish by symmetry.
} 
where the superscript T denotes that these coefficients come from Timoshenko theory. The lowest-order coefficient is, by design, the same as that from Euler-Bernoulli theory $\left(A_{4}^{\mathrm{T}}=A_{4}\right)$, while the next coefficient is $\kappa$-dependent. Requiring (23) and (24) to agree to $q^{6}$ :

$$
A_{6}^{\mathrm{T}}(\kappa)=A_{6},
$$

then allows $\kappa$ to be determined unambiguously since the perturbative calculation invokes no physical assumptions. This settles any remaining controversy $[10,11]$ on the "best" choice for $\kappa$ among those derived by different and slightly ad hoc methods, and puts Timoshenko beam theory, with a canonical choice of the shear coefficient [12-15], on a firm footing.

\section{Conclusion}

We have shown that once the eigenfunction has been found with an error that is $O\left(\varepsilon^{N}\right)$, RQ gives the eigenvalue with an error that is $O\left(\varepsilon^{2 N}\right)$ - a gain of $N$ orders "for free". Such use of RQ, by truncating the eigenfunction but retaining the full $C_{i j k \ell}$ and $\rho$, also ensures positivity for all values of $\varepsilon$, a desirable property. RQ is also more compact than the implicit recursive formulas sometimes used [5,7]. A non-trivial application has been sketched for $N=4$, where a computable 3rd-order eigenfunction gives the 6th-order eigenvalue; this leads to a mathematically unambiguous way of determining the shear coefficient in Timoshenko theory.

\section{Acknowledgements}

D. Kiang drew our attention to Schiff [5] and Löwdin [6]; K.M. Pang drew our attention to Adams [7] and helped to check the Appendix. A. Bhaskar kindly commented on the manuscript.

\section{Appendix A. One-dimensional example}

The theorem is here illustrated with a one-dimensional analog, described by [compare (4)]

$$
-\frac{\mathrm{d}}{\mathrm{d} x}\left\{C(x)\left[\frac{\mathrm{d}}{\mathrm{d} x} \xi(x)\right]\right\}=\lambda \rho(x) \xi(x)
$$

for $x \in \mathcal{V}=[-1,1]$, with $\xi=0$ at the end-points. As an example, take

$$
C(x)=1, \quad \rho(x)=1+2 \varepsilon \delta(x)
$$

This system can be interpreted as the transverse vibration of a string loaded with a point mass $2 \varepsilon$ at the mid-point.

Up to an irrelevant normalization, the solution is

$$
\xi(x)=\sin [q(1-x)] \text { for } x \geq 0,
$$

and symmetric about $x=0$; this is the exact solution for all $\varepsilon$, and different values of $\varepsilon$ only lead to different $q=q(\varepsilon)$, which is determined through the discontinuity of the derivative across $x=0$ to satisfy

$$
\cot q=\varepsilon q
$$

Expanded in powers of $\varepsilon$, the lowest eigenvalue is

$$
q(\varepsilon)=\frac{\pi}{2}\left[1-\varepsilon+\varepsilon^{2}-\left(1-\pi^{2} / 12\right) \varepsilon^{3}+O\left(\varepsilon^{4}\right)\right] .
$$

Truncating this at $N=2$ and putting $q=q(\varepsilon)$ into (A.3) then gives the low-order eigenfunction to be

$$
\xi^{L}=\sin (\pi y / 2)-\varepsilon[(\pi / 2) y \cos (\pi y / 2)] \equiv \xi^{(0)}+\varepsilon \xi^{(1)},
$$

where $y=1-x$. Straightforward evaluation then gives

$$
\begin{gathered}
T\left[\xi^{L}\right]=\int_{-1}^{1} \rho(x) \xi^{L}(x)^{2} \mathrm{~d} x=2 \varepsilon \xi^{L}(x=0)^{2}+2 \int_{0}^{1}\left(\xi^{L}\right)^{2} \mathrm{~d} x \\
=\left[2 \int_{0}^{1}\left(\xi^{(0)}\right)^{2} \mathrm{~d} x\right]+\varepsilon\left[2+4 \int_{0}^{1} \xi^{(0)} \xi^{(1)} \mathrm{d} x\right]+\varepsilon^{2}\left[2 \int_{0}^{1}\left(\xi^{(1)}\right)^{2} \mathrm{~d} x\right] \\
=1+\varepsilon-(1 / 2)\left(1-\pi^{2} / 6\right) \varepsilon^{2} \\
V\left[\xi^{L}\right]=\int_{-1}^{1}\left(\mathrm{~d} \xi^{L} / \mathrm{d} x\right)^{2} \mathrm{~d} x=\left[2 \int_{0}^{1}\left(\mathrm{~d} \xi^{(0)} / \mathrm{d} x\right)^{2} \mathrm{~d} x\right]+\varepsilon\left[4 \int_{0}^{1}\left(\mathrm{~d} \xi^{(0)} / \mathrm{d} x\right)\left(\mathrm{d} \xi^{(1)} / \mathrm{d} x\right) \mathrm{d} x\right]+\varepsilon^{2}\left[2 \int_{0}^{1}\left(\mathrm{~d} \xi^{(1)} / \mathrm{d} x\right)^{2} \mathrm{~d} x\right] \\
=\left(\pi^{2} / 4\right)\left[1-\varepsilon+(1 / 2)\left(1+\pi^{2} / 6\right) \varepsilon^{2}\right],
\end{gathered}
$$




$$
\begin{aligned}
Q\left[\xi^{L}\right] & =\frac{\pi^{2}}{4} \frac{1-\varepsilon+(1 / 2)\left(1+\pi^{2} / 6\right) \varepsilon^{2}}{1+\varepsilon-(1 / 2)\left(1-\pi^{2} / 6\right) \varepsilon^{2}} \\
& \approx \frac{\pi^{2}}{4}\left[1-2 \varepsilon+3 \varepsilon^{2}-\left(4-\pi^{2} / 6\right) \varepsilon^{3}+O\left(\varepsilon^{4}\right)\right],
\end{aligned}
$$

which agrees with the exact $\lambda=q^{2}$ obtained from (A.5) up to the $\varepsilon^{3}$ term [i.e., the error is $O\left(\varepsilon^{4}\right)$ ] —even though $\xi^{L}$ stops at $O(\varepsilon)$ and both the numerator and denominator in (A.7c) stop at $O\left(\varepsilon^{2}\right)$. This example can be carried out to higher orders $N$ without difficulty.

\section{References}

[1] J.W.S. Strutt, (Baron Rayleigh), The Theory of Sound, 1877; reprinted in: J. W. S. Rayleigh, The Theory of Sound, Unabridged Second Revised Edition with an Historical Introduction by R.B. Lindsay, Dover, New York, USA, 1945.

[2] S.S. Rao, Mechanical Vibrations, fourth ed., Pearson Prentice Hall, Upper Saddle River, NJ, USA, 2004.

[3] L. Meirovitch, Principles and Techniques of Vibrations, Prentice Hall, Upper Saddle River, NJ, USA, 1997, p. 238.

[4] C. Pierre, Comments on Rayleigh's quotient and perturbation theory for the eigenvalue problem, Transactions of the ASME Journal of Applied Mechanics 55 (1988) 986-988

[5] L.I. Schiff, Quantum Mechanics, third ed., McGraw-Hill Kogakusha, Tokyo, 1968, See footnote 2 on p. 246 and also an example for $N=2$ in (33.17) on p. 267.

[6] P.-O. Löwdin, Studies in perturbation theory: II. Generalization of the Brillouin-Wigner formalism \& III. Solution of the Schrödinger equation under a variation of a parameter, Journal of Molecular Spectroscopy 13 (1964) 328-337.

[7] B.G. Adams, Algebraic Approach to Simple Quantum Systems: With Applications to Perturbation Theory, Springer-Verlag, Berlin, Germany, 1994, p. 131.

[8] S.P. Timoshenko, On the transverse vibrations of bars of uniform cross-section, Philosophical Magazine 43 (1922) 125-131.

[9] A.E.H. Love, A Treatise on the Mathematical Theory of Elasticity, fourth ed., Dover, New York, 1944, (Chapter XV).

[10] J.D. Renton, A check on the accuracy of Timoshenko beam theory, Journal of Sound and Vibration 245 (2001) $559-561$.

[11] N.G. Stephen, Discussion: A check on the accuracy of Timoshenko beam theory, Journal of Sound and Vibration 257 (2002) $809-812$.

[12] N.G. Stephen, M. Levinson, A second order beam theory, Journal of Sound and Vibration 67 (1979) $293-305$.

[13] N.G. Stephen, Timoshenko's shear coefficient from a beam subjected to gravity loading, ASME Journal of Applied Mechanics 47 (1980) 121-127.

[14] J.R. Hutchinson, Shear coefficient for Timoshenko beam theory, ASME Journal of Applied Mechanics 68 (2001) 87-92.

[15] N.G. Stephen, Discussion: Shear coefficient for Timoshenko beam theory, ASME Journal of Applied Mechanics 68 (2001) 959-960. 